\title{
Post-antibiotic Effect of Various Antibiotics against Achromobacter xylosoxidans Strains Isolated from Patients with Cystic Fibrosis
}

\author{
Damla Damar Çelik ${ }^{1}$, Berna Özbek Çelik ${ }^{1^{*}}$, Niels Nørskov-Lauritsen ${ }^{2}$
} ${ }^{1}$ Istanbul University, Faculty of
Pharmacy, Dept. of Pharmaceutical
Microbiology, Istanbul, Turkey
${ }^{2}$ Department of Clinical
Microbiology, Aarhus University
Hospital Skejby, DK-8200 Aarhus N,
Denmark

\section{Correspondence: \\ Berna Özbek Çelik}

Address: Istanbul University, Faculty of Pharmacy, Dept. of Pharmaceutical Microbiology, Istanbul, Turkey

Email: bernaozbek@hotmail.com

Received: 11.04.2020,

Accepted: 18.05 .2020

https://doi.org/10.5799/jcei/8294

\begin{abstract}
Objectives: Achromobacter xylosoxidans is an emerging pathogen in cystic fibrosis (CF) disease, although relatively limited is known about its clinical impact and optimal management. Pharmacodynamics such as post-antibiotic effect (PAE) are increasingly being applied to the design of antibiotic dosing regimens. In the present study, meropenem, doripenem, colistin, levofloxacin, tigecycline, tobramycin, and chloramphenicol were tested for their PAE.
\end{abstract}

Materials and Methods: The in vitro activities of meropenem, doripenem, colistin, levofloxacin, tigecycline, tobramycin, and chloramphenicol were determined by the microbroth dilution technique. To determine the PAEs, A. xylosoxidans strains in the logarithmic phase of growth were exposed for $1 \mathrm{~h}$ to antibiotics. Recovery periods of test cultures were evaluated using viable counting after centrifugation.

Results: The mean values of in vitro PAEs at $1 \mathrm{xMIC}$ and $4 \mathrm{xMIC}$ concentrations were $3.21 \pm$ 0.66 and $3.58 \pm 0.68$ hours for colistin, $1.54 \pm 0.46$ and $2.27 \pm 0.41$ hours for levofloxacin, 2.34 \pm 0.48 and $3.47 \pm 0.64$ hours for tobramycin, $1.72 \pm 0.47$ and $2.24 \pm 0.41$ hours for tigecycline $1.91 \pm 0.14$ and $2.13 \pm 0.13$ hours for chloramphenicol, respectively. The carbapenem antibiotics exhibited weak PAEs, as expected.

Conclusions: This study's findings could have important implications for the timing of doses during antimicrobial therapy with tested antibiotics.

Keywords: pharmacodynamics, antibiotic, non-fermentative, post-antibiotic effect, Achromobacter xylosoxidans

INTRODUCTION
Achromobacter xylosoxidans, which is an
aerobic, non-fermentative gram-negative
bacillus, is widely distributed in aquatic
environments and has been discovered in
the human ear canal, as well as in the
gastrointestinal and respiratory tracts [1].
The bacterium can cause a variety of
infections, including meningitis,
pneumonia, bacteremia, nosocomial or
urinary tract infections, infection from
contaminated environmental springs such as
dialysis fluids, medications, nebulizers,
disinfectants [2-4]. In particular, the
frequency of diagnosis of
colonization/infection of the airways with $A$. xylosoxidans has raised in cystic fibrosis $(\mathrm{CF})$ patients [5-7]. The prevalence of Achromobacter infections in the group of patients has been observed to differ considerably widely between populations, with estimations of between 16.3 and $23.9 \%$ $[8,9]$. Besides movement tools such as improved swarming capability, which is a flagella-dependent kind of bacterial motility in low-viscosity fluid and aqueous conditions, the main virulence factor of the organism is its capacity to build and then survive within the biofilms [10]. Biofilm product is linked with resistance to environmental agents by improving intimate attachment to surfaces, resistance to phagocytic motion, and other host immune agents, shielding from antimicrobial activity, and improved spread 
across surfaces through bacterial motility. Because $A$. xylosoxidans can assist as a reservoir of horizontal genetic transfer elements usually involved in disseminating antibiotic resistance, the biofilm mode of growth is possible to support the development of multidrug resistance in $\mathrm{CF}$ patients $[11,12]$.

The treatment of Achromobacter infections can be challenging, and the effectiveness of empirical therapy for CF-related infections with commonly available antimicrobials is threatened by the development of microbial resistance [13-16]. Besides the new studies on the urgent need to investigate new antimicrobials against multiresistant bacteria, especially for CF patients $[17,18]$, enhancing management strategies with existing treatments has played a crucial part in identifying dosage schedules for new or old antimicrobials. Therefore, the minimal inhibitory concentration (MIC) and post-antibiotic effect (PAE), which is the delayed regrowth of bacteria following exposure to an antibiotic, were investigated against $A$. xylosoxidans strains isolated from CF patients. In the present study, although antibiotics recommended as first or second or combinations antibiotics category for the treatment of $A$. xylosoxidans were chosen, without PAE antibiotics like beta-lactam antibiotics other than carbapenems and trimethoprim-sulbactam have not been included in this study $[19,20]$.

\section{MATERIALS AND METHODS}

Bacterial Isolates: Four non-epidemiological-related clinical isolates of A. xylosoxidans strains isolated from CF patients and one type strain (LMG $1863^{\mathrm{T}}$ ) were obtained from the Department of Clinical Microbiology, Aarhus University Hospital, Aarhus, Denmark. Identification, $n r d A$ allele and PFGE profile of clinical strains have been reported (AX-1, CF28; AX-2, CF38; AX-3, CF-29; AX-4, CF43) [21]. The Pseudomonas aeruginosa ATTC 27853 reference strain was used to verify that MIC values were within the accuracy range stated by The Clinical and Laboratory Standards Institute guidelines (CLSI) [22].

Antibiotics: Meropenem trihydrate (AstraZeneca Pharmaceuticals, UK), doripenem (Shionogi \& Co. Ltd., Japan), colistin sulfate (Sigma-Aldrich, USA), levofloxacin (Sanofi Pharmaceuticals Inc, Turkey), tigecycline (Wyeth Pharmaceuticals, Collegeville, Pa., USA) tobramycin (Novartis Pharmaceuticals Corp., Turkey) and chloramphenicol (Deva Pharmaceuticals, Turkey) were used. Stock solutions of tested antibiotics were prepared in a solution of $0.85 \%$ to $0.9 \% \mathrm{NaCl}$ (doripenem and tigecycline) or in water for injection (meropenem, levofloxacin, tobramycin, colistin, and chloramphenicol) according to manufacturer's recommendation at a concentration of 5120 $\mathrm{mg} / \mathrm{L}$. The stock solution of colistin, levofloxacin, and tobramycin were stored frozen at $-80^{\circ} \mathrm{C}$ and were used within six months. Meropenem, doripenem, tigecycline, and chloramphenicol solutions were prepared on the day of use.
Media: Cation adjusted Mueller Hinton Broth (CAMHB) was prepared daily by adding $25 \mathrm{mg}$ calcium per liter to liquid and $12.5 \mathrm{mg}$ magnesium per liter to Mueller Hinton Broth (Oxoid Ltd., Basingstoke, UK). This medium was used for MIC and PAE. Tryptic soy agar (Oxoid Ltd.) was used for colony counts.

Determination of MIC: MICs were determined by the microbroth dilution technique described by the CLSI guidelines [22]. Serial two-fold dilutions ranging from 32 to $0.03 \mathrm{mg} / \mathrm{L}$ for meropenem, doripenem, and colistin, from 256 to 0.25 for levofloxacin, tigecycline tobramycin, and chloramphenicol were prepared in fresh CAMHB 96-well microtiter plates. The inoculum was made with a 4 to $6 \mathrm{~h}$ broth culture. Each bacterial strain was spectrophotometrically adjusted to $\mathrm{OD}_{600}=0.12-0.13$ (corresponding to approximately $1 \times 10^{8} \mathrm{CFU} / \mathrm{mL}$ ), and further diluted in CAMHB to obtain a final concentration of $5 \times 10^{5} \mathrm{CFU} / \mathrm{mL}$ in the test tray. The trays were placed in plastic bags to avoid evaporation, incubated at $37^{\circ} \mathrm{C}$ for 18 20 hours, and visually inspected for growth. CLSI interpretative criteria for susceptibility of $P$. aeruginosa ATCC 27853 were used.

Determination of PAEs: PAEs were determined by a standard viable counting method [23, 24]. Strain-antibiotic mixtures were incubated for one $\mathrm{h}$ to avoid prolonged antibiotic exposure and the consequent complete eradication of the organism. At time zero, $1 \mathrm{ml}$ bacterial inoculum $(\mathrm{OD}=0.036)$ was added to tubes containing $29 \mathrm{ml}$ CAMHB with and without (control) test antibiotics producing approximately $10^{6} \mathrm{CFU} / \mathrm{ml}$ as a final concentration of inoculum in the test tubes. The bacteria were exposed to meropenem, doripenem, colistin, levofloxacin, tigecycline, tobramycin, and chloramphenicol at $1 \times$ MIC or $4 \mathrm{xMIC}$. After incubation for one $h$ in a $37^{\circ} \mathrm{C}$-calibrated shaking water bath, cells were pelleted by centrifugation at $5000 \mathrm{rpm}$ for $10 \mathrm{~min}$. The supernatant was poured off, and the pellets were washed twice in buffered sterile saline $(0.9 \% \mathrm{NaCl})$ before being resuspended in $30 \mathrm{ml}$ of prewarmed CAMHB. Bacterial counts of the tube contents were determined at time 0 , immediately before and after centrifugation, and every hour after centrifugation for 8 hours by spreading on pour plates using 10-fold dilutions in saline as needed. Antimicrobial carryover was checked by the inhibition of colonial growth at the site of the initial streak according to NCCLS guidelines [25]. Plates were examined after incubation for 18-24 hour at $37^{\circ} \mathrm{C}$. The PAE was determined by the equation $\mathrm{PAE}=T-C$, at where $T$ is the time (in hour) required for the viability count in the test strain to increase one $\log _{10}$ above the count observed immediately after removal of the antimicrobial agent, and $C$ is the corresponding time for the control strain to grow $1 \log _{10}$. Experiments were conducted in triplicate. The mean PAE value for each strain was calculated by averaging three study values. 
Post-antibiotic effect of various antibiotics against Achromobacter xylosoxidans strains

Table 1. The MICs of tested antibiotics against five $A$. xylosoxidans strains

\begin{tabular}{cccccccc}
\hline \multirow{2}{*}{ Organisms } & \multicolumn{7}{c}{ MIC (mg/L) } \\
\cline { 2 - 8 } & MER & DOR & CS & LVX & ${ }^{a}$ TGC & TOB & CHL \\
\hline AX-1 & 0.125 & 1 & $\mathbf{4}$ & 2 & $\mathbf{4}$ & $\mathbf{6 4}$ & $\mathbf{1 6}$ \\
\hline AX-2 & 0.125 & 0.25 & 0.5 & 2 & $\mathbf{4}$ & $\mathbf{3 2}$ & $\mathbf{1 6}$ \\
\hline AX-3 & 0.06 & 0.25 & $\mathbf{8}$ & 2 & 2 & $\mathbf{1 2 8}$ & $\mathbf{1 6}$ \\
\hline AX-4 & 0.125 & 0.25 & $\mathbf{4}$ & 1 & $\mathbf{4}$ & $\mathbf{3 2}$ & $\mathbf{3 2}$ \\
\hline AX-5 (LMG 1863') & 0.5 & 1 & 2 & $\mathbf{8}$ & $\mathbf{8}$ & $\mathbf{1 2 8}$ & $\mathbf{3 2}$ \\
\hline PA & 0.5 & 0.5 & 1 & 1 & 2 & 1 & $\mathbf{1 2 8}$
\end{tabular}

MER, Meropenem; DOR, doripenem; CS, colistin sulfate; LVX, levofloxacin; TGC, tigecycline; TOB, tobramycin; CHL, chloramphenicol; PA, P. aeruginosa ATCC 27853 (reference strain); AX: Achromobacter xylosoxidans. Bold was used for emphasizing the resistance or intermediated susceptible.

ote: CLSI breakpoints for $P$. aeruginosa for susceptibility and resistance to meropenem are $\leq 2 \mathrm{mg} / \mathrm{L}$ and $\geq 8 \mathrm{mg} / \mathrm{L}$, for doripenem $\leq 2 \mathrm{mg} / \mathrm{L}$ and $\geq 8 \mathrm{mg} / \mathrm{L}$, for colistin are $\leq 2 \mathrm{mg} / \mathrm{L}$ and $\geq 8 \mathrm{mg} / \mathrm{L}$, for levofloxacin are $\leq 2 \mathrm{mg} / \mathrm{L}$ and $\geq 8 \mathrm{mg} / \mathrm{L}$, for tobramycin are $\leq 4 \mathrm{mg} / \mathrm{L}$ and $\geq 16 \mathrm{mg} / \mathrm{L}$ and for chloramphenicol (for other Non-Enterobacteriaceae) are $\leq 8 \mathrm{mg} / \mathrm{L}$ and $\geq 32 \mathrm{mg} / \mathrm{L}$.

aSince no tigecycline CLSI MIC breakpoints exist for tigecycline to non-fermenting Gram-negative bacteria; we use a susceptibility breakpoint of $\leq 2 \mathrm{mg} / \mathrm{L}$ [54].

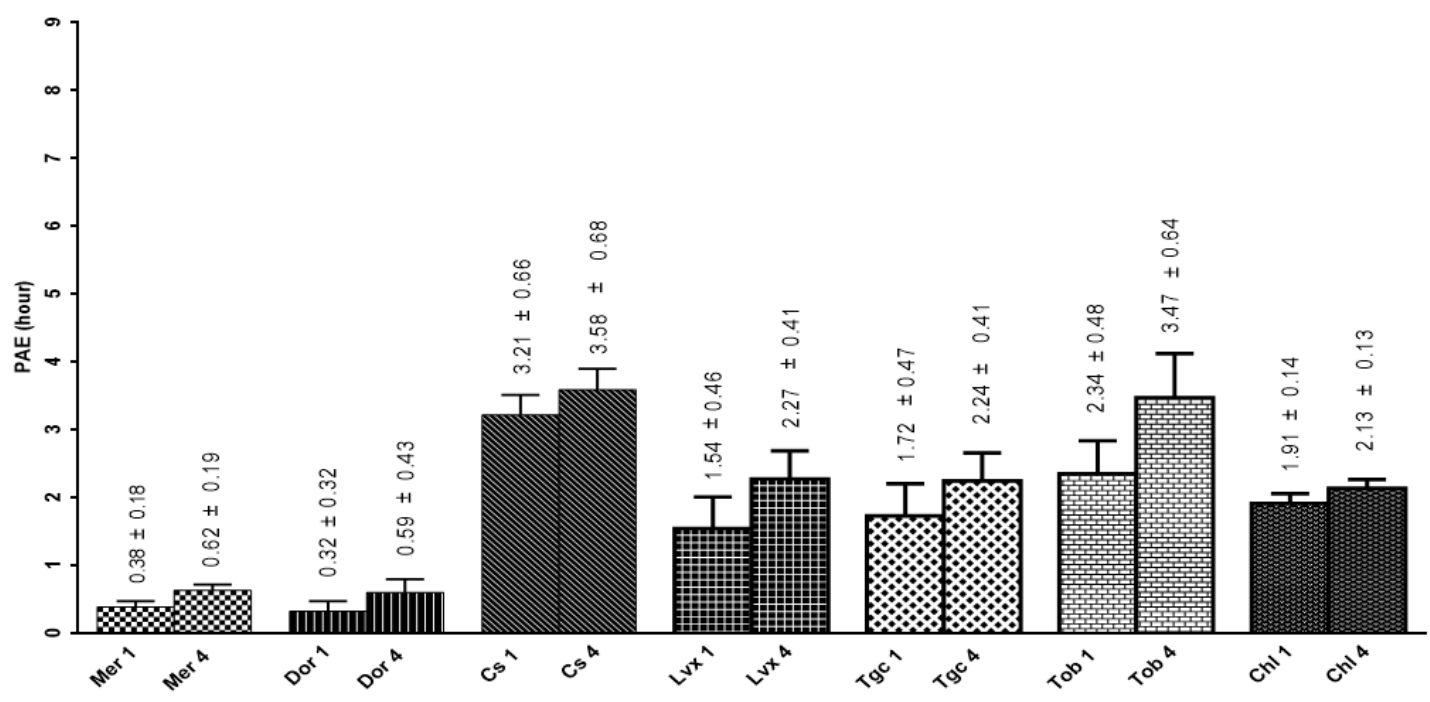

Antibiotics

Figure 1. The mean PAE values of tested antibiotics against 5 A. xylosoxidans strains

Statistical Analysis: Statistical analysis of the study was performed with GraphPad Prism 5.0 (GraphPad Software Inc., San Diego, CA, USA). One-way ANOVA test was used to determine the change in PAE values of each antibiotic concentration. $\mathrm{p}<0.05$ was considered statistically significant.

\section{RESULTS}

The MICs of meropenem, doripenem, colistin, levofloxacin, tigecycline, tobramycin, and chloramphenicol for the five strains of A. xylosoxidans are shown in Table 1. According to the CLSI breakpoint [22], the results revealed that all clinical strains (AX-1, AX-2, AX-3, and AX-4) were susceptible to carbapenems (doripenem and meropenem). AX-2 was susceptible to colistin, whereas AX-3 were resistant. AX-1, AX-2, and AX-4 were resistant to tigecycline, whereas AX-3 was susceptible. The AX-5 strain $\left(\mathrm{LMG} 1863^{\mathrm{T}}\right.$ ) was susceptible to carbapenems and colistin, whereas it was resistant to tigecycline, tobramycin, and levofloxacin. All strain tested were resistant or intermediately resistant to chloramphenicol.

The mean PAE values for 5 A. xylosoxidans after one hour of exposure to antibiotics are displayed in Figure 1, and induction of PAE by meropenem, doripenem, colistin, levofloxacin, tigecycline, tobramycin, and chloramphenicol against each A.xylosoxidans strain is demonstrated in Figure 2. Colistin sulfate produced the longest PAE, ranging from 3.21 to 3.58 hours, although the difference between 1xMIC and $4 \mathrm{xMIC}$ was less than that observed for levofloxacin and tobramycin. Also, remarkable PAEs were induced by tobramycin (ranging from 2.34 to 3.47 hours) $(p<0.001$ ), levofloxacin (ranging from 1.54 to 2.27 hours) $(p<0.01$ ), tigecycline (ranging from 1.72 to 2.24 hours) $(p<0.01$ ), and chloramphenicol (ranging from 1.91 to 2.13 hours) ( $p<$ 0.01 ). On the other hand, carbapenems (meropenem and 

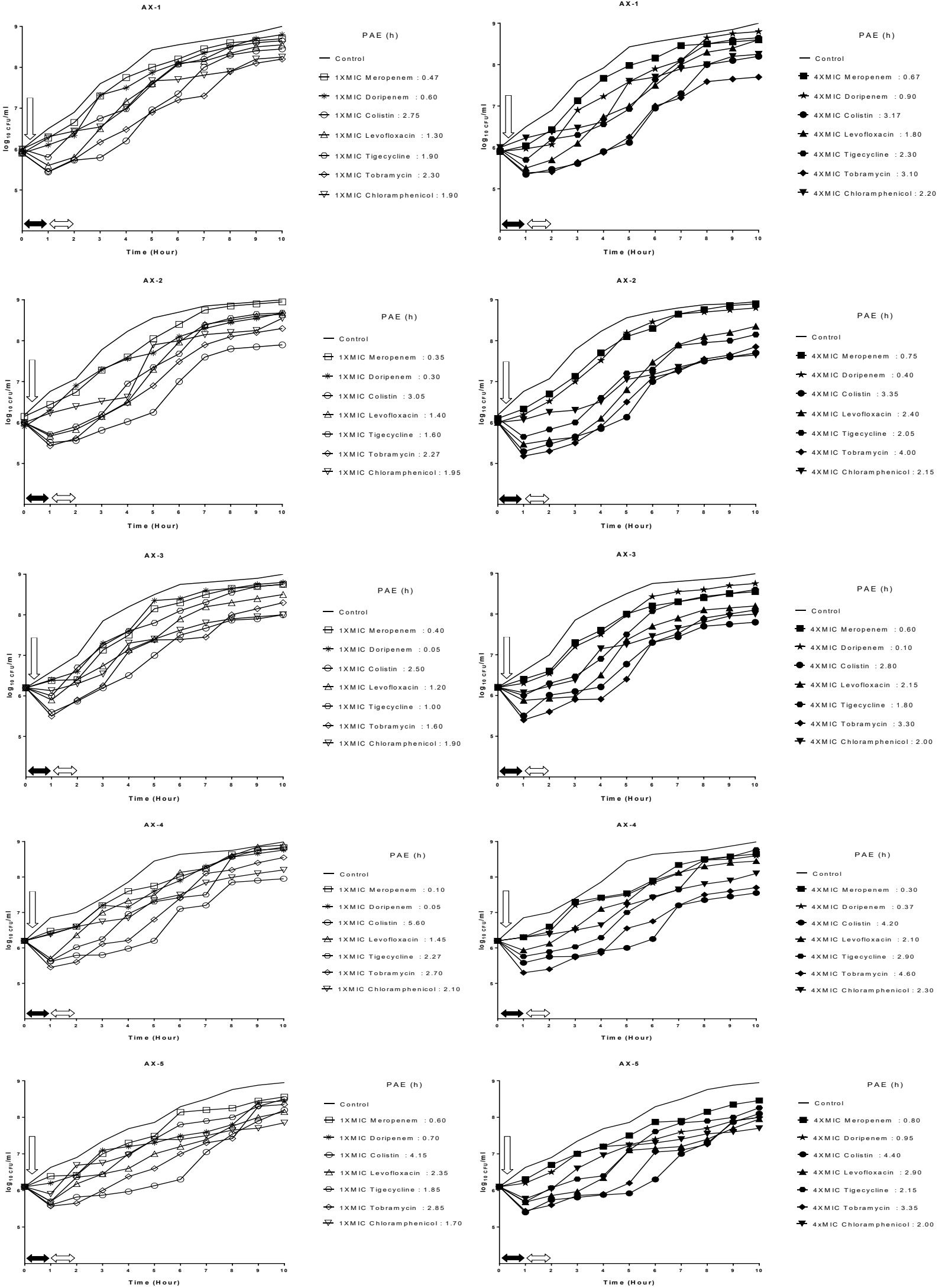

Figure 2. Induction of PAE by meropenem, doripenem, colistin, levofloxacin, tigecycline, tobramycin, and chloramphenicol against $A$. xylosoxidans strains (control, open circles; 1 x MIC, filled circles; $4 \times \mathrm{MIC}$ ). The duration (in hours) of the PAE of each test culture is noted. Timings of the addition and removal of antibiotics and the length of exposure are indicated by the vertical white arrow, the horizontal white arrows, and the horizontal black arrows, respectively 
doripenem) showed modest PAEs (ranging from 0.38 to 0.62 hours and 0.32 o 0.59 hours) against all strains $(p<0.05)$.

\section{DISCUSSION}

PAE is the persistent suppression of bacterial growth after short antimicrobial exposure of antimicrobial agents, and it is increasingly being used to select the most appropriate treatment and dosage schedules [23,25]. A long PAE provides the potential for administering the antimicrobial agent with longer intervals between doses. The PAE appears to be a feature of most antimicrobial agents and has been documented with a variety of common bacterial pathogens [26-28], but not for A. xylosoxidans yet. Several factors influence the presence or duration of the PAE, including the type of organism, type of antimicrobial, concentration of antimicrobial, duration of antimicrobial exposure, and antimicrobial combinations. In vitro methods used to determine the PAE include colony counts, optical density, and measurement of adenosine triphosphate in bacteria. Animal studies reveal in vivo PAEs by PAEs obtained in vitro for most organism/antimicrobial combinations [23,24]. The clinical relevance of the PAE is probably most important when designing dosage regimens. The presence of a long PAE allows aminoglycosides to be dosed infrequently; the lack of an in vivo PAE suggests that beta-lactam antimicrobials require frequent or continuous dosing [23].

Well-defined PAE pharmacodynamic parameters have not yet been firmly established for A. xylosoxidans. In our study, meropenem, which is the first choice carbapenem for A. xylosoxidans antibiotic therapy [19,20,29], was investigated for its PAE. Doripenem, which is unique owing to a sulphamoylaminoethyl-pyrrolidinylthio group at position two resulting in enhanced anti-gram-negative activity [30], offers potentially enhanced carbapenem activity for use in CF, and also might have diminished potential for the emergence of resistance [31], was tested for its PAE. Additionally, previous studies showed that doripenem has similar efficacy to meropenem for patients with pneumonia caused by A. xylosoxidans, and it showed strain-dependent activity with $\mathrm{MIC}_{50}$ and $\mathrm{MIC}_{90}$ of 0.5 and 8 $\mathrm{mg} / \mathrm{L}$ [32-34]. To enhance antibiotics diversity, we performed the in vitro tests, whether doripenem could be a choice for CF patients with A. xylosoxidans pneumonia. Besides, carbapenems are the only $\beta$-lactams with a significant PAE against gram-negative organisms [35-37]. A PAE was observed by Nadler et al. [38] with meropenem when tested against four strains of $P$. aeruginosa (0.8-2 hours) and two strains of Staphylococcus aureus (0.7 and 1.7 hours). Also, a PAE of 1.8 (in vitro) to 4.3 (in vivo) hours for $P$. aeruginosa was recorded for doripenem [34]. Similar to previous studies of other bacteria, modest PAE values were imposed on A. xylosoxidans after 1 hour of exposure to meropenem or doripenem, both at $1 \mathrm{xMIC}$ and $4 \mathrm{xMIC}$.
Colistin sulfate, which has rapid bactericidal activity with a detergent-like mechanism, is a second-line antibiotic in $A$. xylosoxidans therapy $[19,20,29]$. According to our study, it has significant PAEs against the tested A. xylosoxidans strains. It is in agreement with the results on other gramnegative non-fermentative bacteria, such as Acinetobacter baumannii or P. aeruginosa and Enterobacteriaceae such as Klebsiella pneumoniae [27-29]. This antibiotic at 4xMIC alone exhibited more PAEs than 1xMIC. A study showed that patients who received a higher average daily dose of colistin had a lower mortality [39], so the benefit of this prolonged PAE value of colistin sulfate might lead to prolonged dose interval without reduced efficacy, and possibly a lower frequency of adverse events. Clinically, there is not a universally accepted dosing recommendation for colistin. However, the interest in the use of colistin has led to some further understanding of its pharmacokinetics and dosing [40]. Additional studies on pharmacodynamics such as PAE are needed to establish the best colistin therapy protocol for daily dosing.

Tigecycline, owing to its enhanced spectrum of activity and favorable pharmacokinetics, has great potential in the management of Burkholderia cepacia complex (BCC) and several emerging pathogens in CF, namely Stenotrophomonas maltophilia, A. xylosoxidans, and Mycobacterium abscessus [19,20,31]. In vitro studies of tigecycline have demonstrated a prolonged PAE of 8.9 hours for Streptococcus pneumoniae and 4.9 hours for Escherichia coli [41]. Pankuch and Appelbaum [42], measured tigecycline PAEs against 2 A. baumannii strains and, in another previous study [27], reported a PAE value ranging from 0.05 to 1.40 hours against 6 A. xylosoxidans. In the present study, we determined that the PAE of tigecycline lasted 1.72 to 2.24 hours for tested strains of $A$. xylosoxidans. These data indicate the potential for tigecycline to exert a significant PAE at tested concentrations; thus, a long PAE might have an impact on dosing schedules because long PAEs can allow the antibiotic concentration to fall below MIC for significant periods without risk of regrowth.

Levofloxacin is recommended by CLSI as a second-line reporting antibiotic in the therapy of $A$. xylosoxidans $[19,20,29]$. Also, Sader reported that the use of newer fluoroquinolones is preferred when used in combination, given the greater in vitro activity. However, intrinsic resistance and poor activity are widely reported across the class [43]. Furthermore, fluoroquinolones generally produce significant in vitro PAEs against gram-negative strains [4446]. Consistent with previous studies for other gramnegative bacteria, levofloxacin also possesses potent PAEs against A. xylosoxidans, and PAEs were prolonged in a concentration-dependent manner. The presence of a long PAE might allow levofloxacin to be dosed infrequently, perhaps once daily. However, additional studies are needed 
to establish a levofloxacin therapy protocol for once-daily dosing.

Resistance to tobramycin is very high, especially with the intrinsic resistance by enzymatic modification to aminoglycosides, which occurs mainly by aminoglycosides modifying enzymes AAC(6')-Ib and aadA1 [47]. Although this antibiotic is not an option for A. xylosoxidans therapy, previous studies showed synergistic or additive effects for the combination of an aminoglycoside and a $\beta$-lactam against $A$. xylosoxidans strains [48, 49]. Moreover, antimicrobials that inhibit RNA or protein synthesis produce an in vitro PAE against gram-positive cocci and gram-negative bacilli $[23,24]$. Our data show that tobramycin has a significant PAE against $A$. xylosoxidans. These are in agreement with results obtained for other gram-negative and gram-positive bacteria $[23,27,28]$. According to our data, tobramycin PAEs were prolonged in a concentration-dependent manner, and at $4 \mathrm{xMIC}$, tobramycin PAEs was prolonged by approximately $50 \%$ more than when the antibiotic was used at $1 \mathrm{xMIC}$. The very high MIC of this drug plus worrisome side effects will likely prohibit the use of elevated dosing for intravenous administration; however, inhalation of antimicrobials is commonly administered to CF patients. The MICs should be viewed in light of peak sputum concentrations of approximately $1200 \mu \mathrm{g} / \mathrm{g}$ after inhalation of $300 \mathrm{mg}$ tobramycin [50]. The benefit of the prolonged PAE value of tobramycin could justify the use of inhaled tobramycin when intravenous administration is useless.

Chloramphenicol is the first antibiotic to be marketed as the product of chemical synthesis, and it has been produced this way exclusively since 1950 . Chloramphenicol It is a broad-spectrum intravenous (IV) antibiotic [51]. In CF, chloramphenicol is used to treat lung infections caused by specific bacteria - for example, $P$. aeruginosa [52]. In a previous study by Athamna et al. [53] showed that chloramphenicol exhibited a PAEs of 1-2 h for 2 Bacillus anthracis strains at tested MICs. Our in vitro studies of chloramphenicol demonstrated similar PAEs against $A$. xylosoxidans, and PAEs were prolonged in a concentrationdependent manner.

In conclusion, this study shows that the single exposure of colistin, tobramycin, levofloxacin, or tigecycline produces a significant PAE on A. xylosoxidans, which might have implications for the difficult treatment of chronic $A$. xylosoxidans infections. As far as we know, this study is the first investigation of PAEs of various antibiotics that have been used in therapy for infections caused by A. xylosoxidans in CF patients.

Declaration of interest: The authors report no conflicts of interest.

Financial Disclosure: This work was supported by the Research Fund of the University of Istanbul (Istanbul, Turkey). Project number: TYL-2017-23695

\section{REFERENCES}

1. Pickett MJ, Hollis DG, Bottone EJ. Miscellaneous gramnegative bacteria In: Balows A, Hausler WJ Jr, Herrmann KL, Isenberg HD, Shadomy HJ, eds. Manual of clinical microbiology, 5th edn. Washington: DC, 1991:410-28.

2. Gahrn-Hansen B, Alstrup P, Dessau R, et al. Outbreak of infection with Achromobacter xylosoxidans from contaminated intravascular pressure transducers. J Hosp Infect. 1988;12:1-6. doi: 10.1016/01956701(88)90115-6.

3. Stobbelaar K, Van Hoorenbeeck K, Lequesne M, et al. Sepsis Caused by Achromobacter xylosoxidans in a Child with Cystic Fibrosis and Severe Lung Disease. Am J Case Rep. 2016;17:562-6. doi: 10.12659/AJCR.896577.

4. Lambiase A, Catania MR, Del Pezzo M, et al. Achromobacter xylosoxidans respiratory tract infection in cystic fibrosis patients. Eur J Clin Microbiol Infect Dis. 2011;30:973-80. doi: 10.1007/s10096-011-1182-5

5. Spilker T, Vandamme P, Lipuma JJ. Identification and distribution of Achromobacter species in cystic fibrosis. J Cyst Fibros. 2013;12:298-301. doi: 10.1016/j.jcf.2012.10.002

6. Coward A, Kenna DTD, Perry C, et al. Use of nrdA gene sequence clustering to estimate the prevalence of different Achromobacter species among Cystic Fibrosis patients in the UK. J Cyst Fibros. 2016;15:479-85. doi: 10.1016/j.jcf.2015.09.005

7. Spicuzza L, Sciuto C, Vitaliti G, et al. Emerging pathogens in cystic fibrosis: ten years of follow-up in a cohort of patients. Eur J Clin Microbiol Infect Dis. 2009;28:191-5. doi: 10.1007/s10096-008-0605-4.

8. Pereira RHV, Carvalho-Assef AP, Albano RM, et al. Achromobacter xylosoxidans: characterization of strains in Brazilian cystic fibrosis patients. J Clin Microbiol. 2011;49:3649-51. doi: 10.1128/JCM.05283-11.

9. De Baets F, Schelstraete P, Van Daele S, Haerynck F, Vaneechoutte M. Achromobacter xylosoxidans infection in Cystic Fibrosis: prevalence and clinical relevance. J Cyst Fibros. 2007;6(1):75-8. doi: 10.1016/j.jcf.2006.05.011.

10. Pereira RHV, Leao RS, Carvalho-Assef AP, et al. Patterns of virulence factor expression and antimicrobial resistance in Achromobacter xylosoxidans and Achromobacter ruhlandii isolates from patients with cystic fibrosis. Epidemiol Infect. 2017;145:600-6. doi: 10.1017/S0950268816002624.

11. Molin S, Tolker-Nielsen T. Gene transfer occurs with enhanced efficiency in biofilms and induces enhanced stabilization of the biofilm structure. Curr Opin Biotechnol. 2003;14:255-61. doi: 10.1016/S09581669(03)00036-3. 
12. Traglia GM, Almuzara M, Merkier AK, et al. Achromobacter xylosoxidans: an emerging pathogen carrying different elements involved in horizontal genetic transfer. Curr Microbiol. 2012;65:673-8. doi: 10.1007/s00284-012-0213-5.

13. Saiman L, Chen Y, Tabibi S, et al. Identification and antimicrobial susceptibility of Alcaligenes xylosoxidans isolated from patients with cystic fibrosis. J Clin Microbiol. 2001;39:3942-5.

doi: 10.1128/JCM.39.11.3942-3945.2001.

14. Rolston KV, Messer M. The in-vitro susceptibility of Alcaligenes denitrificans subsp. xylosoxidans to 40 antimicrobial agents. J Antimicrob Chemother. 1990;26:857-60. doi: 10.1093/jac/26.6.857.

15. Wang M, Ridderberg W, Hansen CR, et al. Early treatment with inhaled antibiotics postpones next occurrence of Achromobacter in cystic fibrosis. J Cyst Fibros. 2013;12:638-43. doi: 10.1016/j.jcf.2013.04.013.

16. Atalay S, Ece G, Samlioglu P, et al. Clinical and microbiological evaluation of eight patients with isolated Achromobacter xylosoxidans. Scand. J Infect Dis. 2012;44:798-801. doi:10.3109/00365548.2012.664780.

17. Saeed A, Bosch A, Bettiol M, Nossa González DL, Erben MF, Lamberti Y. Novel guanidine compound against multidrug-resistant cystic fibrosis-associated bacterial species. Molecules. 2018;23(5):1158. doi: 10.3390/molecules23051158.

18. Surendran-Nair M, Lau P, Liu Y, Venkitanarayanan K. Efficacy of selenium in controlling Acinetobacter baumannii associated wound infections. Wound Medicine. 2019;26(1):100165. doi: 10.1016/j.wndm.2019.100165.

19. Abbott IJ, Peleg AY. Stenotrophomonas, Achromobacter, and nonmelioid Burkholderia species: antimicrobial resistance and therapeutic strategies. Semin Respir Crit Care Med. 2015;36:99-110. doi: 10.1055/s-0034-1396929

20. Döring G, Flume P, Heijerman H, Elborn JS. Consensus Study Group. Treatment of lung infection in patients with cystic fibrosis: Current and future strategies. J Cyst Fibros. 2012;11:461-479. doi: 10.1016/j.jcf.2012.10.004

21. Gade SS, Nørskov-Lauritsen N, Ridderberg W. Prevalence and species distribution of Achromobacter sp. cultured from cystic fibrosis patients attending the Aarhus centre in Denmark. J Med Microbiol. 2017;66:686-9. doi: 10.1099/jmm.0.000499.

22. Clinical and Laboratory Standards Institute. Performance standards for antimicrobial susceptibility testing: Twenty-fourth informational supplement M100-S24. 2014.

23. Craig WA, Gudmundsson S. The postantibiotic effect. In: Lorian V, ed. Antibiotics in Laboratory Medicine, Baltimore: Williams and Wilkins Inc, 1996:296-329.
24. Bundtzen RW, Gerber AU, Cohn DL, Craig WA. Postantibiotic suppression of bacterial growth. Rev Infect Dis. 1981;3:28-37. doi:10.1093/clinids/3.1.28

25. National Committee for Clinical Laboratory Standards NCCLS. Methods for Determining Bactericidal Activity of Antimicrobial Agents: Approved Guideline M26-A. National Committee for Clinical Laboratory Standards, Wayne: PA, USA. 1999.

26. Ozbek B, Otuk G. Post-antibiotic effect of levofloxacin and tobramycin alone or in combination with cefepime against Pseudomonas aeruginosa. Chemother. 2009;55:446-50. doi: 10.1159/000265128.

27. Ozbek B, Şentürk A. Postantibiotic effects of tigecycline, colistin sulfate, and levofloxacin alone or tigecyclinecolistin sulfate and tigecycline-levofloxacin combinations against Acinetobacter baumannii. Chemother. 2010;56:466-71. doi: 10.1159/000321015.

28. Gaibani P, Lombardo D, Lewis RE, et al. In vitro activity and post-antibiotic effects of colistin in combination with other antimicrobials against colistin-resistant KPCproducing Klebsiella pneumoniae bloodstream isolates. J Antimicrob Chemother. 2014;69:1856-65. doi: 10.1093/jac/dku065.

29. Gibson RL, Burns JL, Ramsey BW. Pathophysiology and management of pulmonary infections in cystic fibrosis. Am J Respir Crit Care Med. 2003;168:918-951. doi: 10.1164/rccm.200304-505SO.

30. Traczewski MM, Brown SD. In vitro activity of doripenem against Pseudomonas aeruginosa and Burkholderia cepacia isolates from both cystic fibrosis and non-cystic fibrosis patients. Antimicrob Agents Chemother. 2006;50:819-21. doi: 10.1128/AAC.50.2.819-821.2006.

31. Parkins MD, Elborn JS. Newer antimicrobial agents and their potential role in cystic fibrosis pulmonary exacerbation management. J Antimicrob Chemother. 2010;65:1853-61. doi: 10.1093/jac/dkq245.

32. Liu WD, Shih MC, Chuang YC, Wang JT, Sheng WH. Comparative efficacy of doripenem versus meropenem for hospital-acquired and ventilator-associated pneumonia. J Microbiol Immunol Infect. 2019;52:78895. doi: 10.1016/j.jmii.2019.04.008.

33. Jones RN, Bell JM, Sader HS, Turnidge JD, Stilwell MG. In vitro potency of doripenem tested against an international collection of rarely isolated bacterial pathogens. Diagn Microbiol Infect Dis. 2009;63:434-9. doi: 10.1016/j.diagmicrobio.2009.01.025.

34. Jones RN, Huynh HK, Biedenbach DJ, Fritsche TR, Sader HS. Doripenem (S-4661), a novel carbapenem: comparative activity against contemporary pathogens including bactericidal action and preliminary in vitro methods evaluations. J Antimicrob Chemother. 2004;54:144-54. doi:10.1093/jac/dkh298. 
35. Bustamante CI, Drusano GL, Tatem BA, Standiford HC Postantibiotic effect of imipenem on Pseudomonas aeruginosa. Antimicrob Agents Chemother. 1984;26:678-82. doi: 10.1128/aac.26.5.678

36. Hanberger S, Vogelman B, Craig WA. The in vivo postantibiotic effect of imipenem and other new antimicrobials. J Antimicrob Chemother. 1986;18:67-73. doi: $10.1093 /$ jac/18.supplement_e.67.

37. Hanberger H, Nilsson LE, Nilsson M, Maller R. Postantibiotic effect of beta-lactam antibiotics on gramnegative bacteria in relation to morphology, initial killing and MIC. Eur J Clin Microbiol Infect Dis. 1991;10:927-34. doi: 10.1007/BF02005446.

38. Nadler HL, Pitkin DH, Sheikh W. The postantibiotic effect of meropenem imipenem on selected bacteria. J Antimicrob Chemoth. 1989;24:225-31. doi: 10.1093/jac/24.suppl_a.225.

39. Li J, Turnidge J, Milne R, Nation RL, Coulthard K. In Vitro Pharmacodynamic Properties of Colistin and Colistin Methanesulfonate against Pseudomonas aeruginosaIsolates from Patients with Cystic Fibrosis. Antimicrob Agents Chemother. 2001;45:781-5. doi: 10.1128/AAC.45.3.781-785.2001.

40. Vicari G, Bauer SR, Neuner EA, Lam SW. Association between colistin dose and microbiologic outcomes in patients with multidrug-resistant gram-negative bacteremia. Clin Infect Dis. 2012;56:398-404. doi: 10.1093/cid/cis909.

41. Van Ogtrop ML, Andes D, Stamstad TJ, et al. In vivo pharmacodynamic activities of two glycylcyclines (GAR-936 and WAY 152,288) against various grampositive and gram-negative bacteria. Antimicrob Agents Chemother. 2000;44:9439. doi: 10.1128/aac.44.4.943949.2000.

42. Pankuch GA, Appelbaum PC. Postantibiotic effect of ceftaroline against Gram-positive organisms. Antimicrob Agents Chemother. 2009;53:4537-9. doi: 10.1128/AAC.00785-09

43. Sader HS, Jones RN. Antimicrobial susceptibility of uncommonly isolated non-enteric Gram-negative bacilli. Int J Antimicrob Agents. 2005;25:95-109. doi: 10.1016/j.ijantimicag.2004.10.002.

44. Houston AK, Jones RN. Postantibiotic effect of DU6859a and levofloxacin as compared with ofloxacin. Diagn Microbiol Infect Dis. 1994;8:57-59. doi: 10.1016/0732-8893(94)90134-1.
45. Minguez F, Ramos C, Barrientos S, Loscos A, Prieto J. Postantibiotic effect of ciprofloxacin compared with that of five other quinolones. Chemother. 1991;7:420-5. doi: $10.1159 / 000238889$.

46. Spangler SK, Bajaksouzian S, Jacobs MR, Appelbaum PC. Postantibiotic effects of grepafloxacin compared to those of five other agents against 12 Gram-positive andnegative bacteria. Antimicrob Agents Chemother. 2000;44:186-9. doi: 10.1128/aac.44.1.186-189.2000.

47. $\mathrm{Hu} \mathrm{Y}, \mathrm{Zhu} \mathrm{Y}, \mathrm{Ma} \mathrm{Y}$, et al. Genomic insights into intrinsic and acquired drug resistance mechanisms in Achromobacter xylosoxidans. Antimicrob Agents Chemother. 2015; 59:1152-61. doi: 10.1128/AAC.0426014.

48. Duggan JM, Goldstein SJ, Chenoweth CE, Kauffman CA, Bradley SF. Achromobacter xylosoxidans Bacteremia: Report of Four Cases and Review of the Literature. Clin Infect Dis. 1996;23:569-576. doi: 10.1093/clinids/23.3.569.

49. Duez JM, Hadjiat-Savioz M, Siebor E, et al. In Vitro Synergistic Activity of Combined Piperacillin and Tobramycin Against Clinical Strains of Achromobacter xylosoxidans. J Chemotherapy. 2010;22:139-41. doi: 10.1179/joc.2010.22.2.139

50. Almuzara M, Limansky A, Ballerini V, et al. In vitro susceptibility of Achromobacter spp. isolates: comparison of disk diffusion, Etest, and agar dilution methods. Int J Antimicrob Agents. 2010;35(1):68-71. doi: 10.1016/j.ijantimicag.2009.08.015.

51. Schwarz S, Kehrenberg C, Doublet B, Cloeckaert A. Molecular basis of bacterial resistance to chloramphenicol and florfenicol. FEMS Microbiol Rev. 2004;28(5):519-42. doi: 10.1016/j.femsre.2004.04.001

52. Morita Y, Tomida J, Kawamura Y. Responses of Pseudomonas aeruginosa to antimicrobials. Front Microbiol. 2014;4:422. doi: 10.3389/fmicb.2013.00422.

53. Athamna A, Athamna M, Medlej B, Bast DJ, Rubinstein E. In vitro post-antibiotic effect of fluoroquinolones, macrolides, beta-lactams, tetracyclines, vancomycin, clindamycin, linezolid, chloramphenicol, quinupristin/dalfopristin and rifampicin on Bacillus anthracis. J Antimicrob Chemother. 2004;53(4):609-15. doi: $10.1093 / \mathrm{jac} / \mathrm{dkh} 130$.

54. Jones RN, Ferraro MJ, Reller LB, et al. Multicenter Studies of Tigecycline Disk Diffusion Susceptibility Results when Testing Acinetobacter spp. J Clin Microbiol. 2006. doi: 10.1128/JCM.01588-06. 